\title{
HCI Practices in Malaysia: A Reflection of ICT Professionals' Perspective
}

\author{
Idyawati Hussein \\ School of Informatics Science \\ Universiti Malaysia Sabah \\ Labuan, F.T., Malaysia \\ idyawati@ums.edu.my
}

\author{
Murni Mahmud \\ Kulliyyah of Information \\ Communication Technology \\ International Islamic University of \\ Malaysia \\ Kuala Lumpur, Malaysia \\ murni@iiu.my
}

\author{
Alvin W. Yeo \\ Faculty of Computer Science and IT \\ Universiti Malaysia Sarawak \\ Kota Samarahan, Malaysia \\ alvin@fit.unimas.my
}

\begin{abstract}
Although Human Computer Interaction (HCI) has been practised by Western countries over the last 40 years, very little is known about how HCI is being incorporated in Malaysian practices. We undertook a 12-week ethnographical study aimed at revealing HCI perceptions at different managerial levels in Information and Communication Technology (ICT) departments and agencies in Malaysia. We describe and discuss the factors that either drive or impede technology managers towards HCI awareness, based on the nature of ICT-related/software development in Malaysia. The result of the study indicates that the developers and corporations' overall perception of $\mathrm{HCI}$ is influenced by their national and organizational culture. The lack of emphasis on usable interface design and scarce information regarding user studies and evaluation are major concerns. Within this context of developing countries, the difficulty of creating $\mathrm{HCI}$ awareness and adopting usability may be due to the complexity of the government's bureaucracy systems. We suggest that stakeholders and policy makers such as the Malaysian Communications and Multimedia Commission (MCMC) and the Malaysian Administrative Modernization and Management Planning Unit (MAMPU) are more relevant in influencing and/or reinforcing the incorporation of $\mathrm{HCI}$ in the workplace and enhancing the usability of the products and software created in the organization at the managerial level.
\end{abstract}

Keywords-HCI, practitioners, government, bureaucracy, Malaysia

\section{INTRODUCTION}

Information and Communication Technology (ICT) is one of the most important determinants of various industries' eventual success in Malaysia. According to the Malaysian Prime Minister, "ICT should be seen as not just an industry to be developed but also as an enabler for major economic sectors to increase productivity and efficiency" [7]. Efficiency means achieving a good fit among the users (who are the citizens), their tasks, and the technology within organizational, social and global contexts [23]. Max Weber noted that work, achievement, and striving for efficiency are important fundamental values. These values and putting them into practive, is key to the success of any society.

In particular, those who are involved in ICT development need to understand why and how people interact with computers in order to accomplish their work and personal goals [21]. Several issues that require attention include: what are the physical, cognitive, affective, and behavioral constraints on the user's side and what pleases or annoys them. It is important to know what makes human-computer interaction a satisfying experience or an experience that users do not want to repeat, and what makes the interaction efficient and effective [6]. This understanding lies in the foundation of Human Computer Interaction (HCI) practice. In order to do gain this understanding, academicians, practitioners, developers and corporations, policy maker in Malaysia need to have a good understanding of important factors that influence the success of $\mathrm{HCI}$ in practice.

HCI is a study of how humans interact with computers, with a focus on understanding how to make computers more useful and usable [21]. It is a discipline concerned with the design, implementation, and evaluation of interactive computing systems for human use. HCI has been around for about 40 years [9]. With such development, is it important to question: what is the current status of HCI in Malaysia? Have the human-computer interfaces been designed well enough to improve work, life and increase productivity? In other countries such as India, they are progressing to institutionalize HCI into their academic and business practices starting from the year 2000 [5]. In Korea, the beginning of $\mathrm{HCI}$ awareness is reflected by the first local HCI conference in 1992, which was affiliated to the ACM Local SIGCHI in Korea [15]. ACM is the Association for Computing Machinery, the oldest organization for professionals in computing.

Although indication on the local's perception and uptake of HCI practice and research can be achieved by looking at the number of HCI-project approved funds at the Ministry of Science level; this study attempt to focus on the current ICT practices. How well HCI is doing in Malaysia seems to be reflected in the awareness [1] of senior IT personnel of the term related to HCI and consider the number of ICT companies having a formal staff position in HCI. The aim of this study is to uncover what is the current status of awareness among practitioners regarding HCI in technologybased companies and agencies among different working sector and job level in Malaysia. This study could be among the first to investigate current HCI status as a beginning to comprehend HCI intend in the System Development Life Cycle (SDLC), thus promoting the utilization of $\mathrm{HCI}$ practices in the Malaysian ICT industry. 